\title{
OPERATORS RELATED TO IDEMPOTENT GENERATED AND MONOID COMPLETELY REGULAR SEMIGROUPS
}

\author{
MARIO PETRICH and NORMAN R. REILLY
}

(Received 3 May 1988; revised 2 March 1989)

Communicated by P. G. Trotter

\begin{abstract}
The class $C R$ of completely regular semigroups (unions of groups or algebras with the associative binary operation of multiplication and the unary operation of inversion subject to the laws $x=x x^{-1} x,\left(x^{-1}\right)^{-1}=x$ and $\left.x x^{-1}=x^{-1} x\right)$ is a variety. Among the important subclasses of $C R$ are the classes $\mathcal{M}$ of monoids and $I$ of idempotent generated members. For each $C \in\{I, \mathcal{M}\}$, there are the associated mappings $\nu \rightarrow V \cap C$ and $\nu \rightarrow\langle\nu \cap C\rangle$, the variety generated by $v \cap C$. The lattice theoretic properties of these mappings and the interactions between these mappings are studied.
\end{abstract}

1980 Mathematics subject classification (Amer. Math. Soc.) (1985 Revision): 20 M 07, 20 M 10.

\section{Introduction and summary}

We consider here completely regular semigroups (unions of groups) as algebras with the binary operation of multiplication and the unary operation of inversion. As such they form a variety to be denoted by $C R$. The operators alluded to in the title of the paper refer to certain operators on the lattice $\mathcal{L}(C R)$ of all subvarieties of $C R$ induced by mappings of the following form. Let $I$ and $\mathcal{M}$ stand for the classes of completely regular semigroups which are idempotent generated or have an identity element, respectively. We consider next mappings on $\mathcal{L}(C R)$ defined by $\mathcal{V} \rightarrow \mathcal{V} \cap I$ and $\mathcal{V} \rightarrow \mathcal{V} \cap M$. The images $\{\mathcal{V} \cap I \mid \mathcal{V} \in \mathcal{L}(C R)\}$ and $\{\mathcal{V} \cap \mathcal{M} \mid \mathcal{V} \in \mathcal{L}(C R)\}$, whose members we dub $I$ - and $\mathcal{M}$-varieties, respectively, are lattices under inclusion. The partitions

(C) 1990 Australian Mathematical Society $0263-6115 / 90 \$ A 2.00+0.00$ 
of $\mathcal{L}(C R)$ induced by these mappings are complete congruences on $\mathcal{L}(C R)$. Considering the ends of the intervals of $\mathcal{L}(C R)$ which constitute the classes of these congruences, we arrive at four operators on the lattice $\mathcal{L}(C R)$.

This paper consists of an investigation in the same general spirit as the authors' article [10] where the classes of $E$-disjunctive and fundamental completely regular semigroups were used in the same setting as are here $I$ and $\mathcal{M}$. These additional mappings give rise to further operators on $\mathcal{L}(C R)$. The semigroups generated by certain sets of these operators induced by the upper ends of the intervals were characterized in authors' paper [9] in terms of generators and relations. The present paper is thus a natural continuation of the two preceding articles on the study of these types of mappings and operators on $\mathcal{L}(C R)$. This work and that of [10] provide a natural setting for them within a general investigation of the structure of $\mathcal{L}(C R)$.

Section 2 contains all the preliminary material needed in the paper. In Section 3 we prove that the mapping $\theta_{I}: \mathcal{V} \rightarrow \mathcal{V} \cap I$ is a complete homomorphism of $\mathcal{L}(C R)$ onto the lattice of $I$-varieties of completely regular semigroups. Its restriction to the lattice of varieties of completely simple semigroups plays an important role in [8]. In the same and the next section, we consider lattice properties of operators associated with the homomorphism $\theta_{I}$. In Section 5 we perform a similar analysis with the class $\mathcal{M}$ of completely regular monoids by considering the mapping $\theta_{\mathcal{M}}: \mathcal{V} \rightarrow \mathcal{V} \cap \mathcal{M}$. The restriction of $\theta_{\mathcal{M}}$ to the lattice of varieties of bands was considered in [13] and [14]. In Section 6 we consider commutativity of the various operators associated with lower ends of intervals. In the final section, Section 7, we calculate the multiplication table for the semigroup generated by two of these operators.

\section{Preliminaries}

If $\theta$ is a mapping of a set $A$, then $\bar{\theta}$ denotes the equivalence on $A$ induced by $\theta$. If also $B \subseteq A$, then $\left.\theta\right|_{B}$ denotes the restriction of $\theta$ to $B$. The equality relation on any set is denoted by $\varepsilon$.

Let $S$ be a completely regular semigroup. For any $a \in S, a^{0}$ denotes the element $a^{-1} a, E(S)$ the set of idempotents of $S, C(S)$, the core of $S$, the subsemigroup of $S$ generated by $E(S)$ and $C(S)$ the lattice of fully invariant congruences on $S$.

We shall use the following notation:

$\tau$-trivial semigroups,

$\mathcal{L} Z$-left zero semigroups,

$R Z$-right zero semigroups, 
$R B$ - rectangular bands,

ReG-rectangular groups,

$S$-semilattices,

$B$-bands,

G-groups,

$S G$-semilattices of groups,

$C S$-completely simple semigroups,

Og-orthogroups (idempotents form a subsemigroup),

$\mathrm{CG}$-cryptogroups ( $H$ is a congruence),

$O C G-O G \cap C G$-orthocryptogroups,

$C R$-completely regular semigroups,

$c(u)$-the set of variables appearing in $u$,

$[u=v]$ - the variety of completely regular semigroups determined

by the identity $u=v$.

The lattice of subvarieties of a variety $\mathcal{V}$ shall be denoted by $\mathcal{L}(\mathcal{V})$. For any $V \in \mathcal{L}(C R)$, we shall denote by $F \mathcal{V}$ the (relatively) free object in $\mathcal{V}$ on $\aleph_{0}$ generators. For a non-empty subclass $A$ of $C R, \mathbf{H} A, \mathbf{S} A, \mathbf{P} A$ and $\langle A\rangle$ shall denote, respectively, the homomorphic closure, the (completely regular) subsemigroup closure, the direct product closure and the variety generated by $A$.

In the first result of this section we give two retractions of sublattices of $\mathcal{L}(C R)$ that will be used later.

LEMMA 2.1. The following mappings are complete endomorphisms:

(i) $\nu \rightarrow V \cap \mathcal{G}(\nu \in \mathcal{L}(C R))$;

(ii) $\nu \rightarrow V \cap C S(\nu \in \mathcal{L}(C R))$.

Proof. Parts (i) and (ii) can be found in [5, Theorems 3.1 and 3.3, respectively].

Let $A$ be a class of completely regular semigroups. Call its members $A$ semigroups. We will say that $A$ is a pre-image class if it is closed under direct products and homomorphic images and has the following property:

for any epimorphism $\theta: S \rightarrow T$, where $S \in C R$ and $T \in$ $A$, there is a completely regular subsemigroup $R$ of $S$ with $R \in A$ and $R \theta=T$.

A subclass $K$ of $A$ is an $A$-variety if it is closed under the formation of direct products, homomorphic images and $A$-subsemigroups. Denote the class of all $A$-varieties by $\mathcal{L}_{A}(C R)$. If $A=C R$, then all $A$-varieties are varieties. 
Proposition 2.2. Let $A$ be a pre-image class of completely regular semigroups.

(i) $\mathcal{L}_{A}(C R)$ is a complete lattice.

(ii) The mapping

$$
\theta_{A}: \mathcal{V} \rightarrow \mathcal{V} \cap A \quad(\mathcal{V} \in \mathcal{L}(C R))
$$

is a complete homomorphism of $\mathcal{L}(C R)$ onto $\mathcal{L}_{A}(C R)$.

Proof. (i) We first show that the mapping $\theta_{A}$ defined in (ii) is a surjection of $\mathcal{L}(C R)$ onto $\mathcal{L}_{A}(C R)$. Since both $\mathcal{V}$ and $A$ are closed under direct products, homomorphic images and $A$-subsemigroups, it follows that $\mathcal{V} \cap A \in \mathcal{L}_{A}(C R)$ and that $\theta_{A}$ maps $\mathcal{L}(C R)$ into $\mathcal{L}_{A}(C R)$. Now let $K \in$ $\mathcal{L}_{A}(C R)$. Since $K$ is closed under the formation of direct products, $\langle K\rangle=$ $\mathbf{H S}(K)$. Let $T \in\langle K\rangle \cap A$. Then there exist $U \in \mathcal{K}$, a completely regular subsemigroup $S$ of $U$ and an epimorphism $\varphi: S \rightarrow T$. By property (P), there exists $R \in A$, which is a completely regular subsemigroup of $S$, such that $R \varphi=T$. But then $R$ is a subsemigroup of $U$ and, since $K$ is closed under $A$-subsemigroups, $R \in K$. Now $K$ is also closed under homomorphic images. Hence $T \in K$. Thus $\langle K\rangle \cap A \subseteq K$. The reverse inclusion is obvious and therefore $K=\langle K\rangle \cap A$. It follows that $\theta_{A}$ is a surjection and from this we can conclude that $\mathcal{L}_{A}(C R)$ is a set.

In addition, it is evident that $\mathcal{L}_{A}(C R)$ is closed under arbitrary intersections and has a greatest element (namely, $A$ ) so that $\mathcal{L}_{A}(C R)$ is, therefore, a complete lattice.

(ii) Clearly $\theta_{A}$ respects arbitrary intersections. In order to show that it is a complete $\bigvee$-homomorphism, we let $\mathcal{V}_{\alpha} \in \mathcal{L}(C R)$, for $\alpha \in A$, and must show that

$$
\left(\bigvee_{\alpha \in A} \mathcal{V}_{\alpha}\right) \cap A=\bigvee_{\alpha \in A}\left(\mathcal{V}_{\alpha} \cap A\right)
$$

Indeed, let $T \in\left(\bigvee_{\alpha \in A} V_{\alpha}\right) \cap A$. Then there exist $V_{\alpha} \in V_{\alpha}$, a subdirect product $S$ of the $V_{\alpha}, \alpha \in A$, and an epimorphism $\theta: S \rightarrow T$. Since $T \in A$ and $A$ has property (P), it follows that there exists a completely regular subsemigroup $R$ of $S$ with $R \in A$ and $R \theta=T$. Then $R$ is a subdirect product of the projections $R_{\alpha}, \alpha \in A$, of $R$ into each $V_{\alpha}$. Since each $R_{\alpha}$ is a homomorphic image of $R$, it follows that $R_{\alpha} \in V_{\alpha} \cap A$ for all $\alpha \in A$. This proves that the left hand side in (1) is contained in the right hand side; the opposite inclusion is trivial. Therefore $\theta_{A}$ is also a complete $V$-homomorphism.

One immediate consequence of Proposition 2.2 is that the class of $A$ varieties is a set. 
The following result of a lattice theoretical nature will be useful.

LEMMA 2.3 [6, Lemma 4.10]. Let $\rho$ be a complete congruence on a complete lattice $L$. For each $x \in L$, let $x^{*}$ be the least element of $x \rho$. Then for any $A \subseteq L$, we have $\bigvee_{\alpha \in A} x^{*}=\left(\bigvee_{\alpha \in A} x\right)^{*}$.

\section{Idempotent generated completely regular semigroups}

Denote by $I$ the class of all idempotent generated completely regular semigroups. For $\mathcal{V} \in \mathcal{L}(C R)$, let

$$
V C=\{S \in C R \mid C(S) \in V\} .
$$

Equivalently, $V C$ consists of those $S \in C R$ all of whose idempotent generated subsemigroups belong to $\mathcal{V}$. Since $\operatorname{HSP}(\nu C)=V C$, as is easily verified, it follows that $\mathcal{V} C \in \mathcal{L}(C R)$. Further let

$$
I=\{C(S) \mid S \in C R\}=\{S \in C R \mid S=C(S)\} .
$$

THEOREM 3.1.

(i) I is a pre-image class.

(ii) The mapping

$$
\theta_{I}: \mathcal{V} \rightarrow \mathcal{V} \cap I \quad(\nu \in \mathcal{L}(C R))
$$

is a complete homomorphism of $\mathcal{L}(C R)$ into $\mathcal{L}_{I}(C R)$. Moreover, for any

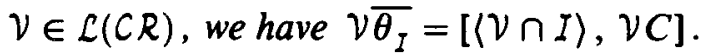

Proof. (i) Let $S \in C R, T \in I$ and $\theta$ be an epimorphism of $S$ onto $T$. For any $e \in E(T)$ and any $a \in S$ such that $a \theta=e$, we have $a^{0} \theta=e$, where $a^{0} \in E(S)$. Since $T$ is idempotent generated, it follows that $C(S) \theta=T$ and that $I$ has property $(\mathbf{P})$. Clearly $I$ is closed under direct products and homomorphic images so that (i) holds.

(ii) It follows immediately from (i) and Proposition 2.2 that $\theta_{I}$ is a complete homomorphism of $\mathcal{L}(C R)$ onto $\mathcal{L}_{I}(C R)$.

Let $\mathcal{V} \in \mathcal{L}(C R)$. It follows easily that

$$
\langle\mathcal{V} \cap I\rangle \cap I=\mathcal{V} \cap I=\mathcal{V} C \cap I,
$$

so that $\langle V \cap I\rangle, \mathcal{V} C \in \mathcal{V} \overline{\theta_{I}}$. Next let $u \in \mathcal{L}(C R)$ be such that $U \cap I=V \cap I$. Then $\langle\mathcal{V} \cap I\rangle=\langle U \cap I\rangle \subseteq U$. Also, for $S \in U$, we have $C(S) \in U \cap I=V \cap I$ so that $S \in \mathcal{V} C$. It follows that $u \subseteq \mathcal{V} C$. Consequently $\mathcal{V} \overline{\theta_{I}}=[\langle\mathcal{V} \cap I\rangle, \mathcal{V} C]$, asserted. 
Corollary 3.2. For any $u, v \in \mathcal{L}(C R)$, we have

$$
U \cap I=\mathcal{V} \cap I \Leftrightarrow\langle U \cap I\rangle=\langle\mathcal{V} \cap I\rangle \Leftrightarrow U C=\mathcal{V} C .
$$

In order to study the mapping $v \rightarrow\langle\nu \cap I\rangle$, we need some preparation.

LEMMA 3.3. The following conditions on $\mathcal{V} \in \mathcal{L}(C R)$ are equivalent.

(i) $v \subseteq O g$.

(ii) $\langle\mathcal{V} \cap I\rangle \subseteq B$.

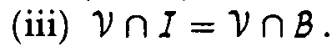

Proof. (i) implies (ii). Clearly $\mathcal{V} \cap I=\mathcal{V} \cap B$ and thus $\langle\mathcal{V} \cap I\rangle=\mathcal{V} \cap B \subseteq B$.

(ii) implies (iii). Evidently $\mathcal{V} \cap I \subseteq\langle\mathcal{V} \cap I\rangle \subseteq \mathcal{V} \cap B$ and the inclusion $\mathcal{V} \cap B \subseteq \mathcal{V} \cap I$ always holds.

(iii) implies (i). If $S \in \mathcal{V}$, then $C(S) \in \mathcal{V} \cap I=\mathcal{V} \cap B$ and hence $S \in O \mathcal{G}$. Therefore $\mathcal{V} \subseteq \mathcal{O G}$.

LEMMA 3.4. The mapping

$$
\mathcal{V} \rightarrow \mathcal{V} \cap B \quad(\mathcal{V} \in \mathcal{L}(O \mathcal{G}))
$$

is a complete endomorphism of $\mathcal{L}(\bigcirc \mathcal{G})$.

Proof. Let $\left\{\mathcal{V}_{\alpha}\right\}_{\alpha \in A}$ be a family of varieties of orthogroups and let $S \in$ $\left(V_{\alpha \in A} V_{\alpha}\right) \cap B$. For each $\alpha \in A$, there exists $V_{\alpha} \in V_{\alpha}$, a subdirect product $P$ of the $V_{\alpha}, \alpha \in A$, and a homomorphism $\varphi$ of $P$ onto $S$. Then $E(P)$ is a subsemigroup of $E\left(\prod_{\alpha \in A} V_{\alpha}\right) \cong \prod_{\alpha \in A} E\left(V_{\alpha}\right)$ and hence $E(P)$ is a subdirect product of its projections $P_{\alpha}$ in the product $\prod_{\alpha \in A} V_{\alpha}$. Note that $P_{\alpha} \in \mathcal{V}_{\alpha} \cap B$ for each $\alpha \in A$. Furthermore, since $S \in B$, the restriction $\left.\varphi\right|_{E(P)}$ is a homomorphism of $E(P)$ onto $S$. Consequently $S \in \bigvee_{\alpha \in A}\left(V_{\alpha} \cap B\right)$. This proves the inclusion $\left(\bigvee_{\alpha \in A} \nu_{\alpha}\right) \cap B \subseteq \bigvee_{\alpha \in A}\left(V_{\alpha} \cap B\right)$; the opposite inclusion is obvious. Since the above mapping is trivially a complete $\bigcap$-homomorphism, the assertion of the lemma follows.

We are now ready to give some properties of the mapping related to the lower bounds of the intervals $v \overline{\theta_{I}}$.

THEOREM 3.5. The mapping

$$
\mathcal{V} \rightarrow\langle\mathcal{V} \cap I\rangle \quad(\mathcal{V} \in \mathcal{L}(C R))
$$

is a complete $V$-endomorphism of $\mathcal{L}(C R)$ but is not an $\bigcap$-homomorphism. Its restriction to $\mathcal{L}(\mathcal{O G})$ is a complete endomorphism of $\mathcal{L}(\mathcal{O G})$.

Proof. The first assertion follows directly from Theorem 3.1 and Lemma 2.3. 
In [4, Example 4.10] an instance of a variety $u$ of completely simple semigroups is given with the following properties:

(i) every $\nVdash$-class of the core of every member of $U$ is generated by elements of order 3;

(ii) there exists a member of $U$ with an element of order 2 in its core.

Let $\mathcal{V}$ be the variety of completely simple semigroups over abelian subgroups of exponent 2 . Then $Z_{2} \in\langle U \cap I\rangle \cap\langle V \cap I\rangle$. On the other hand, let $G \in\langle U \cap \mathcal{V} \cap I\rangle \cap \mathcal{G}$. Since $U \cap \mathcal{V} \cap I$ is closed under direct products, there must exist $Q \in U \cap \mathcal{V} \cap I$, a completely regular subsemigroup $P$ of $Q$ and an epimorphism $\theta: P \rightarrow G$. If $e=1 \theta^{-1}$, where 1 is the identity of $G$, then $H=e P e$ is a subgroup of $Q$ and $\left.\theta\right|_{H}$ is an epimorphism of $H$ onto $G$. Now every $\not$-class of $Q$ is abelian (since $Q \in \mathcal{V}$ ) and generated by elements of order 3 (since $Q \in U \cap I$ ) and so must be of exponent 3. Consequently $Z_{2} \notin\langle U \cap \mathcal{V} \cap I\rangle \cap \mathcal{G}$ so that the mapping $\mathcal{V} \rightarrow\langle V \cap I\rangle$ does not respect intersections. This establishes the second claim of the theorem.

The final assertion of the theorem follows from Lemmas 3.3 and 3.4.

Some of the mappings discussed in this section were considered in [8] for varieties of completely simple semigroups. In particular [8, Proposition

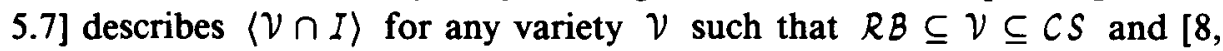
Theorem $7.5(\mathrm{iii})]$ asserts that $C \overline{\theta_{I}}=[A, D]$. Here, for completely simple semigroups, we use the following notation:

A-maximal subgroups are abelian,

$C$-products of idempotents are in the centre of the containing subgroup,

$D$-maximal subgroups of the core are abelian.

\section{Operator $C$}

We are aiming here at an analogue of Theorem 3.5 for the operator $C$ and some supplementary results. Let

$$
\begin{aligned}
\operatorname{Re} B & =\left[a^{0}=a, \text { axya } a x a y a\right], \\
\operatorname{ROG} & =\left[a^{0} b^{0}=\left(a^{0} b^{0}\right)^{0}, a x^{0} y^{0} a=a x^{0} a^{0} y^{0} a\right]
\end{aligned}
$$

denote the varieties of regular bands and regular orthogroups, respectively. The main result of this section is the following, the second assertion of which answers a question posed in [11, page 29].

THEOREM 4.1. The operator $C$ is a complete ก-endomorphism of $\mathcal{L}(C R)$ 
but is not a $\bigvee$-homomorphism. Its restriction to $\mathcal{L}(R \bigcirc \mathcal{G})$ is a complete endomorphism of $\mathcal{L}(R \bigcirc \mathcal{G})$.

Proof. The first assertion follows directly from Theorem 3.1 and the dual of Lemma 2.3; see also [11, Result 7.2(2)]. The proof of the second assertion needs some preparation.

For the ensuing discussion, let $X=\left\{x_{i} \mid i \in I\right\}$ be a countably infinite set, fix $1 \in I$ and let $I^{\prime}=I \backslash\{1\}$. Let

$$
Z=\left\{q_{i} \mid i \in I\right\} \cup\left\{p_{j k} \mid j, k \in I^{\prime}\right\},
$$

$F_{Z}$ be the free group on $Z$ and let $P=\left(p_{j k}\right)$ with $p_{1 k}=p_{k 1}=1$, the identity of $F_{Z}$. Then the Rees matrix semigroup

$$
F C S=\mathcal{M}\left(I, F_{Z}, I ; P\right)
$$

is a free completely simple semigroup over $X$ with embedding $x_{i} \rightarrow\left(i, q_{i}, i\right)$, see [1, Theorem 7.4] and [12, Theorem 1]. In addition, let $F_{q}$ and $F_{p}$ denote the subgroups of $F_{Z}$ (freely) generated by $\left\{q_{i} \mid i \in I\right\}$ and $\left\{p_{j k} \mid j, k \in I^{\prime}\right\}$, respectively. For any normal subgroup $N$ of $F_{Z}$, the relation $\rho_{N}$ defined by

$$
(i, g, j) \rho_{N}(k, h, l) \Leftrightarrow i=k, g h^{-1} \in N, j=l,
$$

is a congruence on $F C S$ with $\rho_{N} \subseteq \nVdash$.

LEMma 4.2 [12, Theorem 3]. (i) There exists a family of endomorphisms $\mathcal{E}$ of $F_{Z}$ such that, for any normal subgroup $N$ of $F_{Z}, \rho_{N}$ is a fully invariant congruence on $F C S$ if and only if $N \in \mathcal{N}$, the lattice of $\mathcal{E}$-invariant normal subgroups of $F_{Z}$.

(ii) If, in the usual anti-isomorphism between the lattice of subvarieties of $C S$ and fully invariant congruences on $F C S, \nu \leftrightarrow \rho_{\nu}$, then for all $\nu \in$ [RB, CS] there exists $N_{\nu} \in \mathcal{N}$ such that $\rho_{\nu}=\rho_{N_{\nu}}$. Moreover, the mapping $\mathcal{V} \rightarrow N_{\mathcal{V}}$ is an anti-isomorphism of $[R B, C S]$ onto $\mathcal{N}$.

For any $M \in \mathcal{N}$, let

$$
M_{q}=M \cap F_{q}, \quad M_{p}=M \cap F_{p}
$$

and for any subgroup $N$ of $F_{Z}$, let $N^{\wedge}$ denote the normal subgroup of $F_{Z}$ generated by $N$.

Lemma 4.3 [8, Proposition 5.1]. For any $v \in[R B, C S]$, with $N=N_{v}$, we have $N_{\nu C}=N_{p}^{\wedge}$. 
Let $u, v \in[R B, C S], N_{u}=M$ and $N_{v}=N$. Then

$$
\begin{aligned}
u C \vee v C=(u \vee v) C & \Leftrightarrow N_{u C} \cap N_{\nu C}=N_{(u \vee \nu) C} \\
& \Leftrightarrow M_{p}^{\wedge} \cap N_{p}^{\wedge}=(M \cap N)_{p}^{\wedge}=\left(M_{p} \cap N_{p}\right)^{\wedge} .
\end{aligned}
$$

Therefore, in order to show that $C$ does not respect joins it suffices to find $M, N \in \mathcal{N}$ such that

$$
M_{p}^{\wedge} \cap N_{p}^{\wedge} \neq\left(M_{p} \cap N_{p}\right)^{\wedge}
$$

Let $M$ and $N$ be the fully invariant subgroups of $F_{Z}$ corresponding to the varieties of abelian groups of exponent 2 and exponent 3, respectively. Then $M_{p}, N_{p}$ and $M_{p} \cap N_{p}$ are the fully invariant subgroups of $F_{p}$ corresponding to the varieties of abelian groups of exponents 2,3 and 6 respectively. We will show that (2) holds.

Let $\alpha \in S_{9}$, the symmetric group on $\{1, \ldots, 9\}$, be the 6-cycle

$$
\alpha=\left(\begin{array}{llllll}
1 & 2 & 3 & 4 & 5 & 6
\end{array}\right) \text {. }
$$

Let $A$ and $B$ be the normal subgroups of $S_{9}$ generated by $\alpha^{2}$ and $\alpha^{3}$, respectively. Now $\alpha^{3}=(14)(25)(36)$ which is an odd permutation. Since the only non-trivial normal subgroups of $S_{9}$ are $A_{9}$, the alternating subgroup, and $S_{9}$ itself, we must have $B=S_{9}$. Moreover $\alpha^{2}=\left(\begin{array}{lll}1 & 3 & 5\end{array}\right)\left(\begin{array}{ll}2 & 4\end{array}\right)$ and so, with $\beta=\left(\begin{array}{ll}1 & 7\end{array}\right)\left(\begin{array}{ll}3 & 8\end{array}\right)\left(\begin{array}{l}5 \\ 5\end{array}\right)$ we obtain

$$
\begin{aligned}
& {\left[\alpha^{2}, \beta\right]=\alpha^{-2} \beta^{-1} \alpha^{2} \beta} \\
& =\left(\begin{array}{lll}
1 & 5 & 3
\end{array}\right)(264)(17)\left(\begin{array}{l}
3 \\
8
\end{array}\right)(59)(135)(246)(17)\left(\begin{array}{ll}
3 & 8
\end{array}\right)(59) \\
& =\left(\begin{array}{lll}
1 & 5 & 3
\end{array}\right)\left(\begin{array}{lll}
7 & 8 & 9
\end{array}\right) \text {. }
\end{aligned}
$$

Let $\gamma=\left[\alpha^{2}, \beta\right]$. Then

$$
\begin{aligned}
& \alpha^{3} \gamma=\left(\begin{array}{lll}
14)(25)(36)(153)(789
\end{array}\right)
\end{aligned}
$$

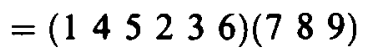

while

$$
\begin{aligned}
& \gamma \alpha^{3}=\left(\begin{array}{lll}
1 & 5 & 3
\end{array}\right)(789)(14)(25)(36) \\
& =\left(\begin{array}{llllll}
1 & 2 & 5 & 6 & 3 & 4
\end{array}\right)\left(\begin{array}{lll}
7 & 8 & 9
\end{array}\right)
\end{aligned}
$$

so that $\alpha^{3} \gamma \neq \gamma \alpha^{3}$ and therefore $\left[\gamma, \alpha^{3}\right] \neq \varepsilon$, the identity of $S_{9}$.

Now let $\theta: Z \rightarrow S_{9}$ be any surjection such that $p_{j k} \theta=\alpha$ for all $j, k \in I^{\prime}$. Then $\theta$ extends to a unique epimorphism of $F_{Z}$ onto $S_{9}$ which we will also denote by $\theta$. Then $F_{p} \theta=\langle\alpha\rangle$, a cyclic group of order 6 . Hence $M_{p} \cap N_{p} \subseteq \operatorname{ker} \theta$ and therefore $\left(M_{p} \cap N_{p}\right)^{\wedge} \subseteq \operatorname{ker} \theta$. 
Let $p=p_{j k}$ for some $j, k \in I^{\prime}$, and let $x=q_{i}$ be such that $x \theta=\beta$. Then $p^{2} \in M_{p}$ so that

$$
a=\left[p^{2}, x\right]=p^{-2}\left(x^{-1} p^{2} x\right) \in M_{p}^{\wedge} .
$$

On the other hand $b=p^{3} \in N_{p} \subseteq N_{p}^{\wedge}$. Hence

$$
[a, b]=\left(a^{-1} b^{-1} a\right) b=a^{-1}\left(b^{-1} a b\right) \in M_{p}^{\wedge} \cap N_{p}^{\wedge} .
$$

However,

$$
a \theta=\left[p^{2}, x\right] \theta=\left[(p \theta)^{2}, x \theta\right]=\left[\alpha^{2}, \beta\right]=\gamma
$$

and $b \theta=p^{3} \theta=\alpha^{3}$ so that

$$
[a, b] \theta=[a \theta, b \theta]=\left[\gamma, a^{3}\right] \neq \varepsilon .
$$

Consequently $[a, b] \notin \operatorname{ker} \theta$ and therefore $[a, b] \in M_{p}^{\wedge} \cap N_{p}^{\wedge}$ but $[a, b] \notin$ $M_{p} \cap N_{p}$. Thus (2) holds and $C$ Is not a $\bigvee$-homomorphism on $\mathcal{L}(C S)$.

This proves the second assertion of Theorem 4.1; we now turn to the proof of the last assertion. The first lemma below shows that the action of $C$ on $\mathcal{L}(O \mathcal{G})$ is really determined by its action on $\mathcal{L}(B)$.

Lemma 4.4. Let $v \in \mathcal{L}(O \mathcal{G})$. Then

$$
\mathcal{V} C=(\mathcal{V} \cap B) C=\{S \in O \mathcal{G} \mid E(S) \in \mathcal{V} \cap B\} .
$$

Proof. We have

$$
\begin{aligned}
\mathcal{V} C & =\{S \in C R \mid C(S) \in \mathcal{V}\} \\
& =\{S \in C R \mid C(S) \in \mathcal{V} \cap B\} \quad \text { since } \mathcal{V} \subseteq O \mathcal{G} \\
& =(\mathcal{V} \cap B) C
\end{aligned}
$$

while clearly

$$
\{S \in C R \mid C(S) \in \mathcal{V} \cap B\}=\{S \in \mathcal{O G} \mid E(S) \in \mathcal{V} \cap B\} .
$$

LEMMA 4.5. Let $\nu \in \mathcal{L}(O \mathcal{G})$ and

$$
\nu \cap B=\left[x^{0}=x, u\left(x_{1}, \ldots, x_{n}\right)=v\left(x_{1}, \ldots, x_{n}\right)\right]
$$

Then

$$
\mathcal{V} C=\left[x^{0} y^{0}=\left(x^{0} y^{0}\right)^{0}, u\left(x_{1}^{0}, \ldots, x_{n}^{0}\right)=v\left(x_{1}^{0}, \ldots, x_{n}^{0}\right)\right] .
$$

Proof. By Lemma 4.4, we have

$$
\mathcal{V} C=\{S \in O \mathcal{G} \mid E(S) \in \mathcal{V} \cap B\}
$$

and clearly, for $S \in O \mathcal{G}, E(S)$ satisfies the identity $u=v$ if and only if $S$ satisfies the identity $u\left(x_{1}^{0}, \ldots, x_{n}^{0}\right)=v\left(x_{1}^{0}, \ldots, x_{n}^{0}\right)$. 
LEMMA 4.6. The restriction of $C$ to $\mathcal{L}(R e B)$ is a complete monomorphism of $\mathcal{L}(\operatorname{Re} B)$ into $\mathcal{L}(R \circ \mathcal{G})$.

Proof. The lattice of subvarieties of $R e \mathscr{B}$ is displayed in Figure 1. From Lemma 4.5 we can determine a basis of identities for each of the varieties $V C(V \in \mathcal{L}(\operatorname{ReB}))$ and can then, with the help of [2, Section 10], identify these varieties in [2, Figure 8.5]. We indicate what this image is for each of the subvarieties of $R e B$ in parentheses in Figure 1 with the obvious interpretation of the abbreviations used. By inspection of [2, Figure 8.5] it can be seen that $\{\nu C \mid \mathcal{V} \in \mathcal{L}(R \in B)\}$ is a sublattice of $\mathcal{L}(R \circ \mathcal{G})$ and therefore that $\mathcal{V} \rightarrow \mathcal{V} C$ is a complete monomorphism.

LEMMA 4.7. The restriction of $C$ to $\mathcal{L}(R O \mathcal{G})$ is a complete $V$-endomorphism.

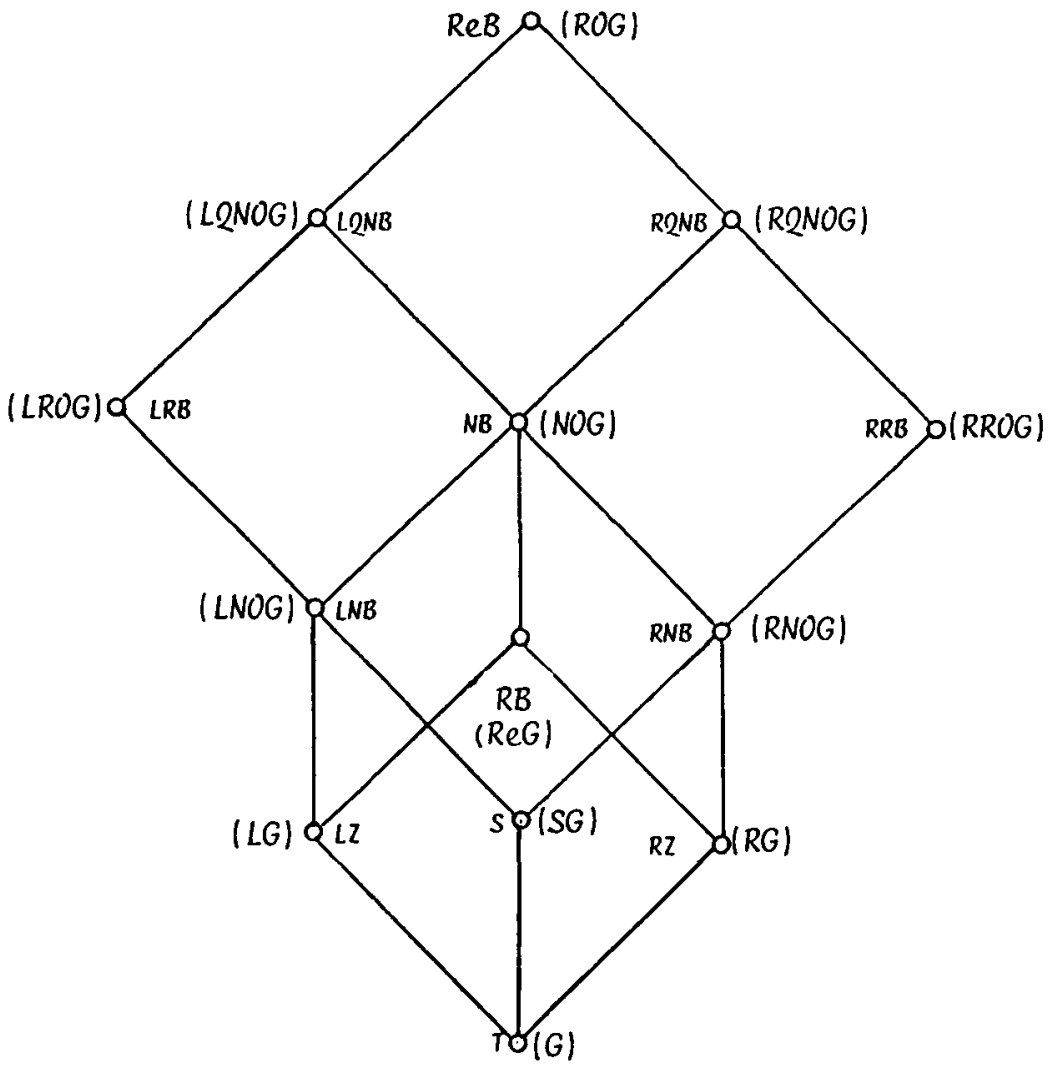

Figure .1. Varieties of regular bands 
Proof. For $\mathcal{V}_{\alpha} \in \mathcal{L}(R \bigcirc \mathcal{G}), \alpha \in A$, we get

$$
\begin{array}{rlrl}
\left(\bigvee_{\alpha \in A} \nu_{\alpha}\right) C & =\left(\left(\bigvee_{\alpha \in A} \nu_{\alpha}\right) \cap B\right) C & \text { by Lemma } 4.4 \\
& =\left(\bigvee_{\alpha \in A}\left(\mathcal{V}_{\alpha} \cap B\right)\right) C & & \text { by Lemma } 3.4 \\
& =\bigvee_{\alpha \in A}\left(\mathcal{V}_{\alpha} \cap B\right) C & & \text { by Lemma 4.6 } \\
& =\bigvee_{\alpha \in A} \nu_{\alpha} C & & \text { by Lemma 4.4. }
\end{array}
$$

This concludes the proof of Theorem 4.1.

We supplement the preceding discussion by showing that the operator $C$ is also an endomorphism when it is restricted to the context of $O C \mathcal{G}=O \mathcal{G} \cap \mathcal{G}$. In other words, we are going to consider the mapping

$$
C^{*}: \mathcal{V} \rightarrow \mathcal{V} C \cap \text { OCg } \quad(\nu \in \mathcal{L}(\text { OCg }))
$$

First we recall

Lemma 4.8 [7, Theorem]. The mapping

$$
\nu \rightarrow(\nu \cap B, \nu \cap \mathcal{G}) \quad(\nu \in \mathcal{L}(O \mathcal{O G}))
$$

is an isomorphism of $\mathcal{L}(\mathcal{O C G})$ onto $\mathcal{L}(B) \times \mathcal{L}(\mathcal{G})$ with inverse $(\mathcal{U}, \mathcal{V}) \rightarrow \mathcal{U V} \mathcal{V}$.

LEMMA 4.9. For any $U \in \mathcal{L}(B)$ and $\mathcal{V} \in \mathcal{L}(\mathcal{G})$, we have $(U \vee \mathcal{V}) C^{*}=U \vee \mathcal{G}$.

Proof. Let $\mathcal{W}=(u \vee v) C^{*}$ and $S \in \mathcal{W} \cap B$. Then $S \in(U \vee v) C \cap B$ so that $S=C(S) \in(U \vee \mathcal{V}) \cap B$. By Lemma 3.4,

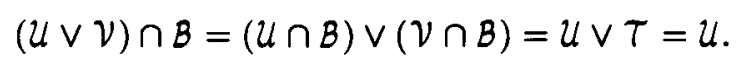

Thus $W \cap B \subseteq U$ and so $W \cap B=U$ since the reverse inclusion is obvious. Also

$$
\tau C^{*}=\tau C \cap O C g=\mathcal{G} \cap O C g=\mathcal{G}
$$

so that $\mathcal{G} \subseteq \mathcal{W}$ and $\mathcal{W} \cap \mathcal{G}=\mathcal{G}$. Applying Lemma 4.8 we have $w=U \vee \mathcal{G}$ and the result follows.

We bring these observations together in the next result. 
Proposition 4.10. The mapping $C^{*}$ is a complete endomorphism of $\mathcal{L}($ OCg)

Proof. For $u_{\alpha} \in \mathcal{L}(O C \mathcal{G})$ with $\alpha \in A$, we have

$$
\begin{aligned}
\left(\bigvee_{\alpha \in A} U_{\alpha}\right) C^{*} & =\left(\bigvee_{\alpha \in A}\left(U_{\alpha} \cap B\right) \vee\left(U_{\alpha} \cap \mathcal{G}\right)\right) C^{*} \\
& =\left[\left(\bigvee_{\alpha \in A}\left(U_{\alpha} \cap B\right)\right) \vee\left(\bigvee_{\alpha \in A}\left(U_{\alpha} \cap \mathcal{G}\right)\right)\right] C^{*} \\
& =\left(\bigvee_{\alpha \in A}\left(U_{\alpha} \cap B\right)\right) \vee \mathcal{G} \text { by Lemma } 4.9 \\
& =\bigvee_{\alpha \in A}\left(\left(U_{\alpha} \cap B\right) \vee \mathcal{G}\right) \\
& =\bigvee_{\alpha \in A} U_{\alpha} C^{*} \text { by Lemma 4.9. }
\end{aligned}
$$

The result now follows by Theorem 4.1 .

\section{Completely regular monoids}

Denote by $\mathcal{M}$ the class of all completely regular monoids. For $\mathcal{V} \in \mathcal{L}(C R)$, let

$$
\mathcal{V} L=\{S \in C R \mid e S e \in \mathcal{V} \text { for all } e \in E(S)\} .
$$

Equivalently, $V L$ consists of those $S \in C R$ all of whose submonoids are in $V$. It is easily verified that $V L \in \mathcal{L}(C R)$. For more information on the operator $L$ see [11, Section 6], where the notation $P$ is used.

The main result of this section is the following.

THEOREM 5.1. (i) $\mathcal{M}$ is a pre-image class.

(ii) The mapping

$$
\theta_{\mathcal{M}}: \nu \rightarrow \nu \cap \mathcal{M} \quad(\nu \in \mathcal{L}(C R))
$$

is a complete homomorphism of $\mathcal{L}(C R)$ onto $\mathcal{L}_{\mathcal{M}}(C R)$. Moreover, for any

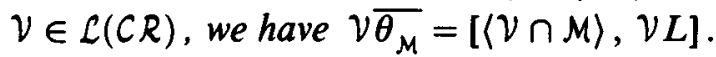

Proof. (i) Clearly $M$ is closed under direct products and homomorphic images. Now let $S \in C R, T \in \mathcal{M}$ and $\theta$ be an epimorphism of $S$ onto $T$. Let $a \in S$ be such that $a \theta=1$, the identity of $T$, and $R=a^{0} S a^{0}$. Then $R \in \mathcal{M}$ and $R \theta=T$. Thus $M$ has the property (P) and (i) holds. 
(ii) That $\theta_{\mathcal{M}}$ is a complete homomorphism of $\mathcal{L}(C R)$ onto $\mathcal{L}_{\mathcal{M}}(C R)$ follows immediately from part (i) and Proposition 2.2.

Let $v \in \mathcal{L}(C R)$. It is easily shown that

$$
\langle\mathcal{V} \cap \mathcal{M}\rangle \cap \mathcal{M}=\mathcal{V} \cap \mathcal{M}=V L \cap \mathcal{M},
$$

so that $\langle\mathcal{V} \cap \mathcal{M}\rangle, \nu L \in \mathcal{V} \overline{\theta_{\mathcal{M}}}$. Next let $U \in \mathcal{L}(C R)$ be such that $U \cap \mathcal{M}=\mathcal{V} \cap \mathcal{M}$. Then

$$
\langle\mathcal{V} \cap \mathcal{M}\rangle=\langle U \cap \mathcal{M}\rangle \subseteq U \text {. }
$$

Also, for $S \in \mathcal{U}$, we have $e S e \in U \cap \mathcal{M}=\mathcal{V} \cap \mathcal{M}$ for all $e \in E(S)$ so that $S \in \mathcal{V} L$. It follows that $u \subseteq \mathcal{V} L$. Consequently $\nu \overline{\theta_{\mathcal{M}}}=[\langle\mathcal{V} \cap \mathcal{M}\rangle, \nu L]$, as asserted.

Corollary 5.2. For any $u, v \in \mathcal{L}(C R)$, we have

$$
u \cap \mathcal{M}=\mathcal{V} \cap \mathcal{M} \Leftrightarrow\langle U \cap \mathcal{M}\rangle=\langle\mathcal{V} \cap \mathcal{M}\rangle \Leftrightarrow U L=V L .
$$

THEOREM 5.3. The mapping

$$
\mathcal{V} \rightarrow\langle\mathcal{V} \cap \mathcal{M}\rangle \quad(\mathcal{V} \in \mathcal{L}(C R))
$$

is a complete endomorphism of $\mathcal{L}(C R)$.

Proof. That the mapping is a complete join endomorphism follows directly from Theorem 5.1 and Lemma 2.3. Let $u_{\alpha} \in \mathcal{L}(C R), \alpha \in A$, and $S \in \bigcap_{\alpha \in A}\left\langle U_{\alpha} \cap \mathcal{M}\right\rangle$. Then $S \in \bigcap_{\alpha \in A} U_{\alpha}$ so that if $S$ has an identity, then

$$
s \in\left(\bigcap_{\alpha \in A} u_{\alpha}\right) \cap \mathcal{M} \subseteq\left\langle\left(\bigcap_{\alpha \in A} u_{\alpha}\right) \cap \mathcal{M}\right\rangle \text {. }
$$

Thus suppose that $S$ does not have an identity and let $S^{1}$ denote $S$ with an identity adjoined. Then, for each $\alpha \in A$, there exist $U_{\alpha} \in U_{\alpha} \cap \mathcal{M}$, a (completely regular) subsemigroup $R_{\alpha}$ of $U_{\alpha}$ and an epimorphism $\theta_{\alpha}: R_{\alpha} \rightarrow$ $S$. Since $S$ does not have an identity neither does $R_{\alpha}$. But $R_{\alpha}^{1}=R_{\alpha} \cup\{1\}$, where 1 is the identity of $U_{\alpha}$, is a subsemigroup of $U_{\alpha}^{\alpha}$ and we can extend $\theta_{\alpha}$ to an epimorphism $\theta_{\alpha}^{\prime}: R_{\alpha}^{1} \rightarrow S^{1}$ by defining $1 \theta_{\alpha}^{\prime}=1$. Thus $S^{1} \in \mathcal{U}_{\alpha} \cap \mathcal{M}$, for all $\alpha \in A$, that is, $S^{1} \in\left(\bigcap_{\alpha \in A} u_{\alpha}\right) \cap \mathcal{M}$ and therefore

$$
s \in\left\langle\left(\bigcap_{a \in A} u_{\alpha}\right) \cap \mathcal{M}\right\rangle \text {. }
$$

Consequently $\bigcap_{\alpha \in A}\left\langle u_{\alpha} \cap \mathcal{M}\right\rangle \subseteq\left\langle\left(\cap_{\alpha \in A} u_{\alpha}\right) \cap \mathcal{M}\right\rangle$ and, since the reverse containment obviously holds, we have equality. 
Our next goal is to indicate how to obtain a basis of identities for $\langle\mathcal{V} \cap \mathcal{M}\rangle$ from one for $\mathcal{V}$. If $\mathcal{V} \in \mathcal{L}(C S)$, then $\mathcal{V} \cap \mathcal{M}=\langle\mathcal{V} \cap \mathcal{M}\rangle=\mathcal{V} \cap \mathcal{G}$. The problem being trivial in this case, we restrict our attention to non-completely simple varieties.

Proposition 5.4. Let $v=\left[u_{\alpha}=v_{\alpha}\right]_{\alpha \in A} \in \mathcal{L}(C R)$, where $c\left(u_{\alpha}\right)=c\left(v_{\alpha}\right)$ for all $\alpha \in A$. For each $\alpha \in A$, let $M_{\alpha}$ be the set of identities obtained from $u_{\alpha}=v_{\alpha}$ by deleting all occurrences of some subset (including the empty subset) of variables. Then $S \in\langle\mathcal{M} \cap\rangle$ if and only if $S$ satisfies all identities of $M_{\alpha}$ for all $\alpha \in A$.

Proof. Direct part. Let $S \in\langle\mathcal{V} \cap \mathcal{M}\rangle$. Since $\mathcal{V} \cap \mathcal{M}$ is closed under direct products, there exists $M \in V \cap M$, a (completely regular) subsemigroup $T$ of $M$ and an epimorphism of $T$ onto $S$. Let $u=v$ be a member of $M_{\alpha}$. Then $M$ satisfies $u=v$ since $M$ satisfies $u_{\alpha}=v_{\alpha}$ and we may substitute the variables in $u_{\alpha}=v_{\alpha}$ which are missing in $u=v$ by the identity of $M$. Hence $T$ and thus also $S$ satisfies $u=v$.

Converse. The given condition implies that $S^{1}$ satisfies $u_{\alpha}=v_{\alpha}$ for every $\alpha \in A$. Hence $S^{1} \in \mathcal{V} \cap \mathcal{M}$ whence $S \in\langle\mathcal{V} \cap \mathcal{M}\rangle$, as required.

Proposition 5.5. The following conditions on $\nu=\left[u_{\alpha}=v_{\alpha}\right]_{\alpha \in A} \in$ $\mathcal{L}(C R)$, where $c\left(u_{\alpha}\right)=c\left(v_{\alpha}\right)$ for all $\alpha \in A$, are equivalent.

(i) $\langle\mathcal{V} \cap \mathcal{M}\rangle=\mathcal{V}$.

(ii) If $S \in \mathcal{V}$, then $S^{1} \in \mathcal{V}$.

(iii) Every $S \in \mathcal{V}$ satisfies all identities which can be obtained from some $u_{\alpha}=v_{\alpha}$ by the deletion of all occurrences of a subset (possibly empty) of variables.

Proof. (i) implies (ii). Let $S \in \mathcal{V}$. Then $S \in\langle\mathcal{V} \cap \mathcal{M}\rangle$ and hence there exist $M \in V \cap M$, a (completely regular) subsemigroup $T$ of $M$ and a homomorphism $\varphi$ of $T$ onto $S$, since $\mathcal{V} \cap \mathcal{M}$ is closed under direct products. Assume that $S$ does not have an identity. Then $T$ also has no identity. Letting 1 be the identity of $M$, we let $T^{\prime}=T \cup\{1\}$ so that $T^{\prime}$ is a monoid. Adjoin an identity $e$ to $S$ thereby obtaining a monoid $S^{1}$. Now extend $\varphi$ to a homomorphism $\varphi^{\prime}$ of $T^{\prime}$ onto $S^{1}$. It follows that $S^{1} \in\langle\mathcal{V} \cap \mathcal{M}\rangle=\mathcal{V}$, as required.

(ii) implies (iii). Let $u=v$ be such an identity and let $S \in \mathcal{V}$. Then by hypothesis, $S^{1} \in \mathcal{V}$ and $S^{1}$ satisfies $u=v$ since $S^{1}$ satisfies $u_{\alpha}=v_{\alpha}$ and $u=v$ is derived from some such identity by setting some (possibly empty) set of variables equal to 1 . But then $S$ also satisfies $u=v$.

(iii) implies (i). Let $S \in \mathcal{V}$. The hypothesis implies that for every $\alpha \in A$, $S^{1}$ satisfies $u_{\alpha}=v_{\alpha}$ and hence $S^{1} \in \mathcal{V}$. It follows that $S^{1} \in \mathcal{V} \cap \mathcal{M}$ and thus $S \in\langle\mathcal{V} \cap \mathcal{M}\rangle$. Therefore $\mathcal{V} \subseteq\langle\mathcal{V} \cap \mathcal{M}\rangle$ and the opposite inclusion is trivial. 


\section{Commutativity between operators}

In [10] and the previous sections we have introduced various classes of completely regular semigroups and have shown how each determines a decomposition of $\mathcal{L}(C R)$ into certain intervals. Associated with the lower and upper ends of these intervals are various operators on $\mathcal{L}(C R)$ and we devote this section to the study of the interaction of the operators associated with the lower ends. Information about the operators associated with the upper ends can be found in [9].

Let $\rho$ be a congruence on $S \in C R$. Then

$$
\operatorname{ker} \rho=\{a \in S \mid a \rho e \text { for some } e \in E(S)\}
$$

is the kernel of $\rho$ and $\operatorname{tr} \rho=\left.\rho\right|_{E(S)}$ is the trace of $\rho$. The greatest congruence on $S$ for which $\operatorname{ker} \rho=E(S)$ is denoted by $\tau$ and the greatest congruence such that $\operatorname{tr} \rho=\varepsilon$ is denoted by $\mu$. If $\tau=\varepsilon, S$ is $E$-disjunctive, and if $\mu=\varepsilon, S$ is fundamental. For any equivalence relation $\lambda$ on $S, \lambda^{0}$ denotes the largest congruence $\rho$ on $S$ contained in $\lambda$. If $A \subseteq S$, let

$$
A^{0}=((A \times A) \cup(S \backslash A) \times(S \backslash A))^{0} .
$$

In terms of this notation, $\tau=E(S)^{0}$ and $\mu=H^{0}$. If $\mathcal{L}^{0}=\varepsilon$, then $S$ is left fundamental.

The classes considered in [10] were

$$
\begin{aligned}
D & =\{S \in C R \mid S \text { is } E \text {-disjunctive }\}, \\
\mathcal{F} & =\{S \in C R \mid S \text { is fundamental }\}, \\
\mathcal{L} \mathcal{F} & =\{S \in C R \mid S \text { is left fundamental }\} .
\end{aligned}
$$

Taken together with the classes discussed in the preceding sections, this leads us to consider the following operators (all defined on $\mathcal{L}(C R)$ ):

$$
\begin{aligned}
& d: \mathcal{V} \rightarrow\langle\mathcal{V} \cap D\rangle, \quad t: \mathcal{V} \rightarrow\langle\mathcal{V} \cap \mathcal{F}\rangle, \\
& t_{l}: \mathcal{V} \rightarrow\langle\mathcal{V} \cap \mathcal{L} \mathcal{F}), \quad c: \mathcal{V} \rightarrow\langle\mathcal{V} \cap I\rangle, \\
& l: \mathcal{V} \rightarrow\langle\mathcal{V} \cap \mathcal{M}\rangle .
\end{aligned}
$$

In the first result we identify which pairs of the above operators commute with each other. This section consists of the proof of this statement.

THEOREM 6.1.

(i) $d l=l d$. (ii) $t t_{l}=t_{l} t=t_{l}$. (iii) $t c=c t$.

(iv) $t l=l t$. (v) $t_{l} c=c t_{l}$. (vi) $t_{l} l=l t_{l}$.

Proof. Part (i). We require the following lemma. 
LEMMA 6.2 [9, LEMMA 6.1]. Let $S$ be a completely regular semigroup. If $e \in E(S)$, then $\left.\tau_{s}\right|_{e s e}=\tau_{e S e}$.

The proof of part (i) follows easily from the next lemma which, in fact, provides additional information of independent interest.

LEMMA 6.3. For any $v \in \mathcal{L}(C R)$, we have

$$
\langle\langle\nu \cap D\rangle \cap \mathcal{M}\rangle=\langle\langle\nu \cap \mathcal{M}\rangle \cap D\rangle=\langle\nu \cap \mathcal{M} \cap D\rangle \text {. }
$$

Proof. It suffices to prove that

$$
\begin{aligned}
& \langle\mathcal{V} \cap D\rangle \cap \mathcal{M} \subseteq\langle\mathcal{V} \cap D \cap \mathcal{M}\rangle, \\
& \langle\mathcal{V} \cap \mathcal{M}\rangle \cap D \subseteq\langle\mathcal{V} \cap D \cap \mathcal{M}\rangle .
\end{aligned}
$$

Let $S \in\langle\mathcal{V} \cap D\rangle \cap \mathcal{M}$. Since $\mathcal{V} \cap D$ is closed under direct products, there exists $Q \in \mathcal{V} \cap D$, a (completely regular) subsemigroup $P$ of $Q$ and an epimorphism $\varphi: P \rightarrow S$. Denote by 1 the identity of $S$, let $e \in E\left(1 \varphi^{-1}\right)$ and $U=e Q e$. By Lemma 6.2, $\tau_{U}=\left.\tau_{Q}\right|_{e Q e}=\left.\varepsilon\right|_{e Q e}=\varepsilon_{U}$. Therefore $U \in D$. We thus have $U \in \mathcal{V} \cap \mathcal{M} \cap D$ and $S$ is a homomorphic image of the subsemigroup $e P e$ of $U$. Consequently $S \in\langle\mathcal{V} \cap M \cap D\rangle$ which establishes relation (3).

Now let $S \in\langle\mathcal{V} \cap \mathcal{M}\rangle \cap D$. Then there exist $Q \in \mathcal{V} \cap \mathcal{M}$, a (completely regular) subsemigroup $P$ of $Q$ and an epimorphism $\varphi: P \rightarrow S$. If $S$ has an identity, then $S \in \mathcal{V} \cap M \cap D$. So suppose that $S$ does not have an identity. Since $Q$ has an identity, it follows that $P^{1}$ is a subsemigroup of $Q$ so that $P^{1} \in \mathcal{V} \cap \mathcal{M}$ and we can extend $\varphi$ to an epimorphism of $P^{1}$ onto $S^{1}$ by defining $1 \varphi=1$. Suppose that $S^{1} \notin D$. Then there must be a non-trivial congruence $\rho$ on $S^{1}$ with $\operatorname{ker} \rho=E\left(S^{1}\right)$. Hence $\left.\operatorname{ker} \rho\right|_{S}=E(S)$. But $S \in D$. Consequently $\left.\rho\right|_{S}=\varepsilon$ and therefore $\rho$ can have only one nontrivial class, namely $1 \rho$, and that class can contain only two elements, say $1 \rho=\{1, e\}$. Since $\operatorname{ker} \rho=E\left(S^{1}\right)$, we must have $e \in E(S)$. But then $S^{1} / \rho$ contains (an isomorphic copy of) $S$ as a subsemigroup and has $e \rho=1 \rho$ as an identity. Hence $e$ must be an identity for $S$, which is a contradiction. Therefore $S^{1} \in D$ so that $S^{1} \in \mathcal{V} \cap D \cap \mathcal{M} \subseteq\langle\mathcal{V} \cap D \cap \mathcal{M}\rangle$ and (4) holds.

Part (ii). For the claim that $t t_{l}=t_{l} t=t_{l}$, we wish to establish, for any $\mathcal{V} \in \mathcal{L}(C R)$, the following equalities:

$$
\langle\langle\mathcal{V} \cap \mathcal{F}\rangle \cap \mathcal{L} \mathcal{F}\rangle=\langle\langle\mathcal{V} \cap \mathcal{L} \mathcal{F}\rangle \cap \mathcal{F}\rangle=\langle\mathcal{V} \cap \mathcal{L} \mathcal{F}\rangle .
$$

Clearly $\mathcal{L} \mathcal{F} \subseteq \mathcal{F}$ and $\langle\mathcal{V} \cap \mathcal{F}\rangle \cap \mathcal{F}=\mathcal{V} \cap \mathcal{F}$. Hence

$$
\langle\mathcal{V} \cap \mathcal{F}\rangle \cap \mathcal{L} \mathcal{F}=\langle\mathcal{V} \cap \mathcal{F}\rangle \cap \mathcal{F} \cap \mathcal{L F}=\mathcal{V} \cap \mathcal{F} \cap \mathcal{L F}=\mathcal{V} \cap \mathcal{L F}
$$


and the first and third classes in (5) are equal. Further,

$$
\mathcal{V} \cap \mathcal{L} \mathcal{F}=\langle\mathcal{V} \cap \mathcal{L F}\rangle \cap \mathcal{L} \mathcal{F} \subseteq\langle\mathcal{V} \cap \mathcal{L} \mathcal{F}\rangle \cap \mathcal{F}
$$

so that $\langle\mathcal{V} \cap \mathcal{L} \mathcal{F}\rangle \subseteq\langle\langle\mathcal{V} \cap \mathcal{L} \mathcal{F}\rangle \cap \mathcal{F}\rangle$. Since the reverse containment is obvious, the second and third classes in (5) are also equal.

Part (iii). We will proceed to the proof of part (iii) via two lemmas, the first of which gathers together the necessary information on $\mu$, the largest congruence contained in $k$.

LEMMA 6.4. Let $S \in C R$.

(i) $a \mu b \Leftrightarrow a^{0}=b^{0}$ and $a^{-1} e a=b^{-1} e b$ for all $e \leq a^{0}$.

(ii) If $S$ is fundamental and $e \in E(S)$, then eSe is also fundamental.

(iii) If $\varphi: S \rightarrow T$ is an epimorphism and $a, b \in S$ are such that $a \mu b$, then $a \varphi \mu b \varphi$.

(iv) $S / \mu$ is fundamental.

Proof. See [3, Theorem 5].

In the next lemma we shall require the following concept. For any $\rho \in$ $C(S), S \in C R$ and any completely regular subsemigroup $P$ of $S$, the saturation $P^{\prime}$ of $P$ by $\rho$ is $P^{\prime}=\bigcup_{p \in P} p \rho$. Clearly $P^{\prime}$ is also a completely regular subsemigroup of $S$.

LEMMA 6.5. For any $\nu \in \mathcal{L}(C R)$, we have

$$
\langle\langle\mathcal{V} \cap I\rangle \cap \mathcal{F}\rangle=\langle\langle\mathcal{V} \cap \mathcal{F}\rangle \cap I\rangle=\langle\mathcal{V} \cap \mathcal{F} \cap I\rangle .
$$

Proof. Letting

$$
A=\langle\mathcal{V} \cap I\rangle \cap \mathcal{F}, \quad B=\langle\mathcal{V} \cap \mathcal{F}\rangle \cap I
$$

and

$$
C=\langle\mathcal{V} \cap \cap I\rangle,
$$

it suffices to prove that $A \subseteq C$ and $B \subseteq C$.

First, let $S \in A$. Since $V \cap I$ is closed under direct produts, there exists $Q \in \mathcal{V} \cap I$, a (completely regular) subsemigroup $P$ of $Q$ and an epimorphism $\varphi: P \rightarrow S$. Let $\mu$ be the greatest idempotent separating congruence on $Q$. Since $Q$ is idempotent generated, so is $Q / \mu$, and by Lemma 6.4(iv), $Q / \mu$ is fundamental. Consequently $Q / \mu \in \mathcal{V} \cap \mp \cap I$. Let $P^{\prime}$ be the saturation of $P$ by the congruence $\mu$ and let $\theta=\left.\mu\right|_{P^{\prime}}$. Then $P^{\prime} / \theta$ is a subsemigroup of $Q / \mu$ so that $P^{\prime} / \theta \in C$. Let $a, b \in P$ be such that $a \theta b$. Since $\theta$ is an idempotent separating congruence on $P^{\prime}$, we have $a \mu^{\prime} b$ where $\mu^{\prime}$ is the 
greatest idempotent separating congruence on $P^{\prime}$. It follows from Lemma 6.4(iii) that $a \varphi$ and $b \varphi$ are related by the greatest idempotent separating congruence on $S$. By hypothesis, $S \in \mathcal{F}$ and therefore $a \varphi=b \varphi$. This makes it possible to define a function $\psi$ by

$$
\psi: a \theta \rightarrow a \varphi \quad(a \in P) .
$$

It follows easily that $\psi$ is an epimorphism of $P^{\prime} / \theta$ onto $S$. Therefore $S \in C$.

Next let $S \in B$. In the above notation, we now assume that $Q \in \mathcal{V} \cap$ $\mathcal{F}$. The expression for the greatest idempotent separating congruence, see Lemma 6.4(i), shows that it depends only on idempotents. It then follows that $\left.\mu\right|_{C(Q)}$ is the greatest idempotent separating congruence on $C(Q)$. Since $Q$ is fundamental, we get that $C(Q)$ is fundamental as well. Thus $C(Q) \in$ $\mathcal{V} \cap \mathcal{F} \cap, C(P)$ is a subsemigroup of $C(Q)$, and $\left.\varphi\right|_{C(P)}$ is a homomorphism of $C(P)$ onto $S$ since $S \in I$. Consequently $S \in C$.

Part (iii) is an immediate consequence of the above lemma.

Part (iv). We wish to show that for any $\mathcal{V} \in \mathcal{L}(C R)$, we have

$$
\langle\langle\mathcal{V} \cap \mathcal{M}\rangle \cap \mathcal{F}\rangle=\langle\langle\mathcal{V} \cap \mathcal{F}\rangle \cap \mathcal{M}\rangle=\langle\mathcal{V} \cap \mathcal{F} \cap \mathcal{M}\rangle \text {. }
$$

Letting $A=\langle\mathcal{V} \cap \mathcal{M}\rangle \cap \mathcal{F}, B=\langle\mathcal{V} \cap \mathcal{F} \cap \mathcal{M}$ and $C=\langle\mathcal{V} \cap \mathcal{F} \cap \mathcal{M}\rangle$, it suffices to prove that $A \subseteq C$ and $B \subseteq C$. Let $S \in A$. Then, since $M$ is closed under direct products, there exist $M \in \mathcal{V} \cap \mathcal{M}$, a (completely regular) subsemigroup $T$ of $M$ and an epimorphism $\varphi: T \rightarrow S$. Now $S \in \mathcal{V}$ since $M \in \mathcal{V}$ and also $S \in \mathcal{F}$ whence $S \in \mathcal{V} \cap \mathcal{F}$. If $S \in \mathcal{M}$, then $S \in \mathcal{V} \cap \mathcal{F} \cap \mathcal{M}$. Assume that $S \notin M$. Then $T \notin \mathcal{M}$; letting 1 be the identity of $M$, we get $T^{1} \subseteq M$. Adjoin an identity $e$ to $S$ and extend $\varphi$ to $T^{1}$ by letting $1 \varphi=e$. Then $S^{1} \in \mathcal{V}$ and also $S^{1} \in \mathcal{F}$. Hence $S^{1} \in \mathcal{C}$ and thus $S \in \mathcal{C}$.

Let $S \in B$. Then there exist $F \in \mathcal{V} \cap \mathcal{F}$, a (completely regular) subsemigroup $T$ of $F$ and an epimorphism $\varphi: T \rightarrow S$. Now $S \in \mathcal{V}$ since $F \in \mathcal{V}$ and also $S \in \mathcal{M}$ whence $S \in \mathcal{V} \cap \mathcal{M}$.

Let 1 be the identity of $S$ and let $e \in E(T)$ be such that $e \varphi=1$. Then $(e T e) \varphi=S, e T e$ is a subsemigroup of $e F e$, and $e F e \in \mathcal{F}$ by Lemma 6.4(ii). Hence $e F e \in \mathcal{V} \cap \mathcal{M} \cap \mathcal{F}$ and $S \in \mathcal{C}$.

Part (v). We first gather together various observations about $\mathcal{L}^{0}$ which parallel the observations in Lemma 6.4 for $\mu=\mathcal{H}^{0}$.

LEMMA 6.6. Let $S \in C R$.

(i) If $e \in E(S)$, then $\left.\mathcal{L}_{S}^{0}\right|_{e S e}=\mathcal{L}_{e S e}^{0}$.

(ii) If $S$ is left fundamental and $e \in E(S)$, then eSe is also left fundamental. 
(iii) If $\varphi: S \rightarrow T$ is an epimorphism and $a, b \in S$ are such that $a \mathcal{L}^{0} b$, then $a \varphi \mathcal{L}^{0} b \varphi$.

(iv) $S / \mathcal{L}^{0}$ is left fundamental.

(v) $\mathcal{L}_{S}^{0} \mid C(S)=\mathcal{L}_{C(S)}^{0}$.

(vi) If $S$ is left fundamental, so is $C(S)$.

Proof. (i) See [9, Lemma 6.4].

(ii) This follows directly from part (i).

(iii) This is an immediate consequence of the observation that, for any $a, b \in e S e$,

$$
a \mathcal{L}_{S}^{0} b \Leftrightarrow x a \mathcal{L} x b \text { for all } x \in S^{1},
$$

(iv) See [6, Corollary 5.7].

(v) See [9, Lemma 5.4].

(vi) This follows directly from part (v).

The remainder of the proof of the equality in part $(v)$ of Theorem 6.1 is then entirely analogous to that of part (iii) but using Lemma 6.6 in place of Lemma 6.4.

Part (vi). The proof of this part is entirely similar to that of part (iv).

\section{The semigroup generated by $c$ and $l$}

The operators $c$ and $l$ do not commute; for example $C S c l=\mathcal{G}$ while $c S l c=\tau$. However, it is possible without too much difficulty to determine the semigroup generated by $c$ and $l$. We gather the basic observations that we require in the next lemma.

LEMMA 7.1.

(i) $c^{2}=c, l^{2}=l$.

(ii) $l c l=l c$.

(iii) $\mathcal{V} l c \subseteq \mathcal{V} c l$ for all $\mathcal{V} \in \mathcal{L}(C R)$.

(iv) $c l c=l c$.

Proof. (i) This is obvious from Theorems 3.1 and 5.1.

(ii) Let $\mathcal{V} \in \mathcal{L}(C R)$. From the definition of $l$, it is clear that $v l c l \subseteq \mathcal{V} l c$. In order to establish the reverse inclusion, it suffices to show that $\langle\mathcal{M} \cap\rangle \cap I \subseteq$ $V l c l$. Let $S \in(\mathcal{V} \cap \mathcal{M}\rangle \cap I$. Since $\mathcal{V} \cap \mathcal{M}$ is closed under direct products, there exists $M \in \mathcal{V} \cap \mathcal{M}$, a (completely regular) subsemigroup $R$ of $M$ and an epimorphism $\theta: R \rightarrow S$. Since $S \in I$ and every idempotent in $S$ is the 
image under $\theta$ of an idempotent in $R, \theta$ maps $C(R)$ onto $S$. So, without loss of generality, we may take $R=C(R) \in I$.

If $S$ contains an identity 1 and $e \in E(R)$ is such that $e \theta=1$, then $(e \operatorname{Re}) \theta=S$. Also

$$
e R e \in \mathcal{V} \cap \mathcal{M} \cap I \subseteq\langle\mathcal{V} \cap \mathcal{M}\rangle \cap I
$$

so that $S \in\langle\langle\mathcal{V} \cap \mathcal{M}\rangle \cap I\rangle$ and therefore $S=S^{1} \in \mathcal{V} l c l$.

If $S$ does not contain an identity and 1 is the identity of $M$, then $1 \notin R$. But

$$
R^{1}=R \cup\{1\} \in \mathcal{V} \cap \mathcal{M} \cap I \subseteq\langle\langle\mathcal{V} \cap \mathcal{M}\rangle \cap I\rangle
$$

and $\theta$ extends to an epimorphism of $R^{1}$ onto $S^{1}$. Hence

$$
S^{\prime} \in\langle\langle\mathcal{V} \cap \mathcal{M}\rangle \cap I\rangle \cap \mathcal{M}
$$

and so $S \in \mathcal{V} l c l$. Thus $\mathcal{V} l c \subseteq \mathcal{V} l c l$ and equality prevails.

(iii) By (ii), $\nu l c=v l c l \subseteq \mathcal{V} c l$ for all $\mathcal{V} \in \mathcal{L}(C R)$.

(iv) For all $\mathcal{V} \in \mathcal{L}(C R)$, we have

$$
\begin{aligned}
\nu l c & \supseteq v c l c \\
& \supseteq v l c l \text { by (iii) } \\
& =v l c \quad \text { by }(\mathrm{i})
\end{aligned}
$$

so that $V l c=V c l c$.

That the four operators $l, c, l c$ and $c l$ are all distinct can be seen from the following:

$$
c S c=c s, \quad c s l=c S c l=g l=\mathcal{G}, \quad c s l c=g c l=\tau .
$$

These observations combined with Lemma 7.1 clearly give the following result.

THEOREM 7.2. The semigroup generated by the operators $c$ and $l$ has the following multiplication table.

\begin{tabular}{|c|c|c|c|c|}
\hline & $c$ & $l$ & $c l$ & $l c$ \\
\hline$c$ & $c$ & $c l$ & $c l$ & $l c$ \\
\hline$l$ & $l c$ & $l$ & $l c$ & $l c$ \\
\hline$c l$ & $l c$ & $c l$ & $l c$ & $l c$ \\
\hline$l c$ & $l c$ & $l c$ & $l c$ & $l c$ \\
\hline
\end{tabular}


We conclude this section by demonstrating the non-commutativity of the remaining pairs of operators from the list introduced at the beginning of this section.

1. $d t \neq t d$.

For consider $C S$. If $S \in C S$, then $S$ is a subdirect product of $S / H$ and $S / \tau$. Hence if $S \in C S \backslash \operatorname{Re} \mathcal{G}$, then $S / \tau \notin \mathcal{G}$ for otherwise $S$ would be a subdirect product of a rectangular band and a group. Hence there exists a non-group $T \in C S \cap D$. Thus $T / H$ is a non-trivial rectangular band and $T / H \in\langle C S \cap D\rangle \cap \mp$ so $\langle\langle C S \cap D\rangle \cap F\rangle \neq T$. On the other hand

$$
\langle\langle C S \cap \mathcal{F}\rangle \cap D\rangle=\langle R B \cap D\rangle=\langle\tau\rangle=\tau \text {. }
$$

2. $d t_{l} \neq t_{l} d$.

This is a variant of the above, where $C S \cap \mathcal{L} F=R Z$.

3. $d c \neq c d$.

For consider $S g$. Let $S=Z_{2}^{0}$, where $Z_{2}=\{e, g\}$ is a 2-element group with identity $e$. Let $Y_{2}$ be a 2-element semilattice. Then $e g=g \notin E(S)$ and $0 g=0 \in E(S)$ so that $(e, 0) \notin \tau$ and $Y_{2} \cong S / \mathcal{H}$. Thus $Y_{2} \in\langle S \mathcal{G} \cap D\rangle \cap I$ and $\langle\langle S \mathcal{G} \cap D\rangle \cap I\rangle \neq \tau$. On the other hand,

$$
\langle\langle S \mathcal{G} \cap I\rangle \cap D\rangle=\langle S \cap D\rangle=\langle\tau\rangle=\tau .
$$

\section{References}

[1] A. H. Clifford, 'The free completely regular semigroup on a set', J. Algebra 49 (1979), 434-451.

[2] J. A. Gerhard and M. Petrich, 'All varieties of regular orthogroups', Semigroup Forum 31 (1985), 311-351.

[3] T. E. Hall, 'On regular semigroups', J. Algebra 24 (1973), 1-24.

[4] P. R. Jones, 'Completely simple semigroups: free products, free semigroups and varieties', Proc. Royal Soc. Edinburgh Sect. A 88 (1981), 293-313.

[5] P. R. Jones, 'On the lattice of varieties of completely regular semigroups', J. Austral. Math. Soc. Ser. A 35 (1983), 227-235.

[6] F. Pastijn and M. Petrich, 'Congruences on regular semigroups', Trans. Amer. Math. Soc. 295 (1986), 607-633.

[7] M. Petrich, 'Varieties of orthodox bands of groups', Pacific J. Math. 58 (1975), 209-217.

[8] M. Petrich and N. R. Reilly, 'Near varieties of idempotent generated completely simple semigroups', Algebra Universalis 16 (1983), 83-104.

[9] M. Petrich and N. R. Reilly, 'Semigroups generated by certain operators on varieties of completely regular semigroups', Pacific J. Math. 132 (1988), 151-175.

[10] M. Petrich and N. R. Reilly, 'Operators related to $E$-disjunctive and fundamental completely regular semigroups' (to appear, J. of Algebra).

[11] L. Polák, 'On varieties of completely regular semigroups IIl', Semigroup Forum 37 (1988), 1-30. 
[12] V. V. Rasin, 'Free completely simple semigroups', Ural. Gos. Univ. Mat. Zap. 11 (1979), 140-151 (Russian).

[13] N. R. Reilly, 'Varieties of completely regular semigroups', J. Austral Math. Soc. Ser. A 38 (1985), 372-393.

[14] S. Wismath, 'The lattice of varieties and pseudovarieties of band monoids', Semigroup Forum 33 (1986), 187-198.

\section{Simon Fraser University}

Burnaby, B.C.

Canada 